\title{
Spin-orbit hybrid entanglement of photons and quantum contextuality
}

\author{
Ebrahim Karimi, ${ }^{1,}$ * Jonathan Leach, ${ }^{2}$ Sergei Slussarenko, ${ }^{1}$ Bruno Piccirillo, ${ }^{1,3}$ Lorenzo Marrucci,,${ }^{1,4}$ \\ Lixiang Chen, ${ }^{5}$ Weilong She, ${ }^{5}$ Sonja Franke-Arnold, ${ }^{2}$ Miles J. Padgett, ${ }^{2}$ and Enrico Santamato ${ }^{1,3}$ \\ ${ }^{1}$ Dipartimento di Scienze Fisiche, Università di Napoli "Federico II", \\ Compl. Univ. di Monte S. Angelo, 80126 Napoli, Italy \\ ${ }^{2}$ Department of Physics and Astronomy, University of Glasgow, Glasgow, Scotland, UK \\ ${ }^{3}$ Consorzio Nazionale Interuniversitario per le Scienze Fisiche della Materia, Napoli \\ ${ }^{4}$ CNR-SPIN, Compl. Univ. di Monte S. Angelo, 80126 Napoli, Italy \\ ${ }^{5}$ State Key Laboratory of Optoelectronic Materials and Technologies, \\ Sun Yat-sen University, Guangzhou 510275, China
}

\begin{abstract}
We demonstrate electromagnetic quantum states of single photons and of correlated photon pairs exhibiting "hybrid" entanglement between spin and orbital angular momentum. These states are obtained from entangled photon pairs emitted by spontaneous parametric down conversion, by employing a $q$-plate for coupling the spin and orbital degrees of freedom of a photon. Entanglement and contextual quantum behavior (that is also non-local, in the case of photon pairs) is demonstrated by the reported violation of the Clauser-Horne-Shimony-Holt inequality. In addition a classical analog of the hybrid spin-orbit photonic entanglement is reported and discussed.
\end{abstract}

\section{INTRODUCTION}

Entangled states are at the heart of most quantum paradoxes and provide the main tool for quantum information processing, including applications such as teleportation, cryptography, superdense coding, etc. Entangled quantum states are also the basis of Bell's inequality violations, which ruled out classical hidden-variable theories in favor of quantum mechanics [1]. Bell's inequalities were originally derived for two particles, as a consequence of locality and realism. In almost all experimental demonstrations of these inequalities to date, the same degree of freedom of two particles has been used, e.g. the spin of a photon. Very recently, however, the case of so-called "hybrid entanglement", occurring when the involved degrees of freedom of the two particles are not the same, has attracted a certain interest, and the first experimental demonstrations with spin and spatialmode degrees of freedom have been reported $[2,3]$. Using different degrees of freedom also opens up another opportunity, i.e. that of realizing entanglement between different degrees of freedom of a single particle. In this case, no role is played by non-locality, but Bell-type inequalities can still be formulated by assuming realism and the so-called "non-contextuality" of the two involved commuting observables, i.e. the assumption that the result of a particular measurement of one observable is determined independently of any simultaneous measurement of the other one [4-6]. Non-contextual hidden variable models have been excluded by recent experiments where the violation of suitable inequalities was observed using neutrons [7], ions [8], and single photons prepared in entangled spin-path states [9]. Finally, single-particle entanglement, in the case of bosons such as photons, has a classical analog that is obtained by replacing singlephoton states with multi-photon coherent states realized within the same field mode [10]. Such a classical analog helps visualizing the nature of the single-particle entanglement.

A particularly convenient framework in which to explore these concepts is provided by photons carrying both spin angular momentum (SAM) and orbital angular momentum (OAM). While the former is the most widely employed internal degree of freedom of photons for quantum manipulations, the latter is becoming an interesting additional resource for quantum applications (see, e.g., [11-14]). In this work, we study three conceptually related experimental situations. Firstly, heralded single photons are prepared in a state where SAM and OAM are entangled (as proposed in [15]), and are then used for testing the contextuality of different degrees of freedom of the same particle. Secondly, correlated photon pairs, where the SAM of one photon is entangled with the OAM of the other, i.e. photon pairs exhibiting SAMOAM hybrid entanglement, are generated and used for testing the contextuality and non-locality of these degrees of freedom when they are spatially separated. Finally, optical coherent states involving many photons, are used to demonstrate a classical analog of SAM-OAM hybrid entanglement.

\section{EXPERIMENTAL SETUP}

The experimental layout we used in the quantum regime (the first two experiments) is presented in Fig. 1. Our down-conversion source generates photon pairs that are entangled in the OAM degree of freedom [17, 18], each photon being horizontally polarized, as described by

$$
|\psi\rangle=\sum_{m=-\infty}^{\infty} c_{|m|}|m\rangle_{o}^{A}|-m\rangle_{o}^{B}|H\rangle_{\pi}^{A}|H\rangle_{\pi}^{B} .
$$

Here $A$ and $B$ denote the signal and idler photons traveling along the two corresponding arms of the setup shown 


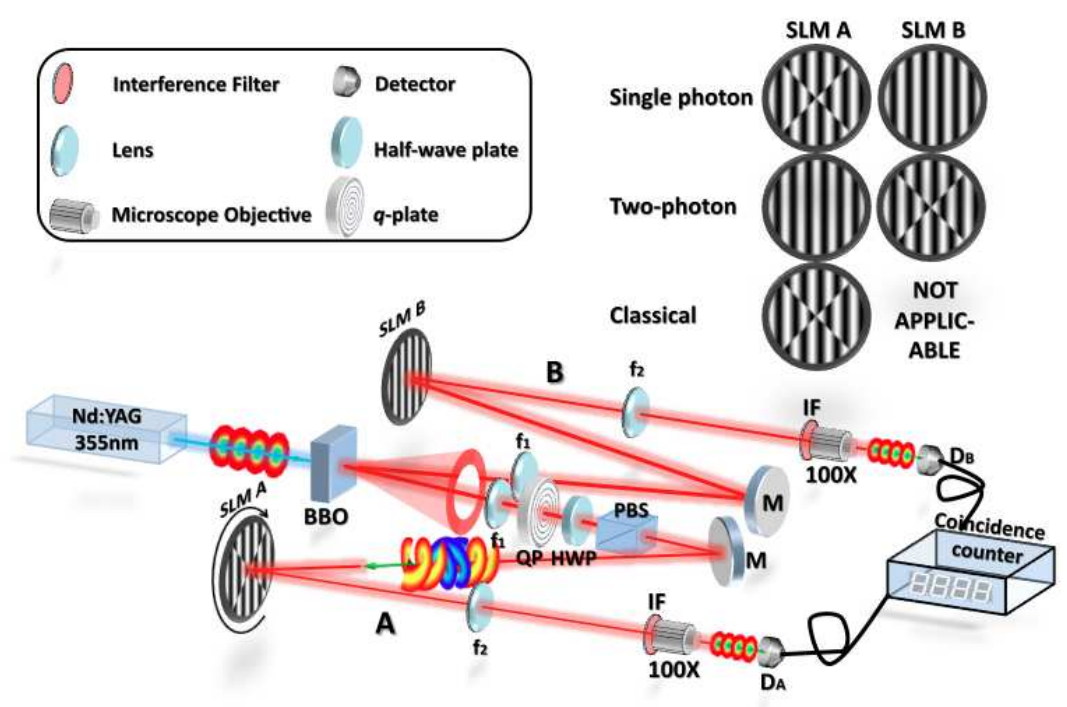

FIG. 1: Setup used for the two quantum-regime experiments. A Nd:YAG laser with average power of $150 \mathrm{~mW}$ at $355 \mathrm{~nm}$ pumps a nonlinear crystal of $\beta$-barium borate (BBO) cut for degenerate type-I non-collinear phase matching which emits OAM-entangled $H$-polarized photon pairs at $710 \mathrm{~nm}$ (see Ref. [18] for details). The photons of each pair are split in arms A and $\mathbf{B}$, respectively. Legend of the main components (see also graphic symbol legend in the upper-left inset): $\mathrm{f}_{1}, \mathrm{f}_{2}-\mathrm{lenses}_{\mathrm{for}}$ beam control; QP - q-plate; HWP - half-wave plate; PBS - polarizer; M - mirror; SLM A and SLM B - spatial light modulators; IF - interference filter for bandwidth definition; 100X - microscope objectives for fiber coupling; $\mathrm{D}_{A}, \mathrm{D}_{B}-\mathrm{photon}$ detectors. In the classical-regime experiment, the optical line is the same as arm A. Top-right inset: computer-generated hologram patterns displayed on the two SLMs in the three experiments.

in Fig. 1 and $\pi$, $o$ denote SAM and OAM degrees of freedom, respectively. The integer $m$ is the photon OAM in units of $\hbar$ and $H$ denotes horizontal linear polarization.

\section{Single-photon experiment}

. In this case we use photon $B$ to herald a single photon $A$ which we convert into an OAM-SAM maximally entangled state. Starting from state $|\psi\rangle$ given in Eq. (1), we post-select photon pairs having $m=0$, i.e. in state $|\psi\rangle=c_{0}|0\rangle_{o}^{A}|0\rangle_{o}^{B}|H\rangle_{\pi}^{A}|H\rangle_{\pi}^{B}$, by coupling photon $B$ into a single-mode optical fiber. Photon $A$ is thus also projected into $m=0$. Spatial light modulator SLM B in this case is patterned as a uniform grating, deflecting the beam but not affecting its transverse spatial mode (see upperright inset of Fig. 1). Photon $A$ is sent first through a $q$-plate $[19,20]$ so as to generate the maximally entangled SAM-OAM state [21]

$$
\left|\Phi^{+}\right\rangle^{A}=\frac{1}{\sqrt{2}}\left(|R\rangle_{\pi}^{A}|+2\rangle_{o}^{A}+|L\rangle_{\pi}^{A}|-2\rangle_{o}^{A}\right)
$$

where $L, R$ denote left-circular and right-circular polarization states, respectively. The polarization state of photon $A$ emerging from the $q$-plate is then measured by a half-wave plate (HWP) oriented at a variable angle $\theta / 2$ and a fixed linear polarizer, restoring the horizontal polarization. This HWP-polarizer combination filters incoming photons having linear polarization at angle $\theta$ with respect to the horizontal direction. In the circular polarization basis, the state of the filtered photons is written as $|\theta\rangle_{\pi}=\frac{1}{\sqrt{2}}\left(e^{i \theta}|L\rangle_{\pi}+e^{-i \theta}|R\rangle_{\pi}\right)$. The SAM measurement does not affect the OAM degree of freedom. Non-contextuality can be assumed between the $z$ component of photon SAM and OAM, because, in the paraxial approximation, the SAM operator $\widehat{S}_{z}$ commutes with the OAM operator $\widehat{L}_{z}$. After SAM filtering, the photon's OAM is also measured by a suitable computergenerated hologram, displayed on SLM A, followed by coupling into a single-mode fiber. The hologram pattern is defined by the four-sector alternated $\pi$-shift phase structure shown in the upper-right inset of Fig. 1, with the four sectors rotated at a variable angle $\chi$ (the grating fringes are not rotated). On diffraction, this hologram transforms the photons arriving in the OAM superposition state $|\chi\rangle_{o}=\frac{1}{\sqrt{2}}\left(e^{2 i \chi}|+2\rangle_{o}+e^{-2 i \chi}|-2\rangle_{o}\right)$ back into the $m=0$ state, which is then filtered by coupling in fiber. The OAM superposition state $|\chi\rangle_{o}$ is the spatial mode analog of the linear polarization, and we may refer to its angle $\chi$ as to its "orientation" [22]. The overall effect of our apparatus is therefore to perform a joint measurement of the polarization and spatial mode orientations of $A$ photons at angles $\theta$ and $\chi$, respectively. When photon $A$ is in the entangled Bell state described by Eq. (2), we expect that the final probability to detect 
it (in coincidence with the $B$ trigger photon) is given by

$$
\left.P(\theta, \chi)=\left|{ }^{A}\left\langle\Phi^{+}|\cdot| \theta\right\rangle_{\pi}^{A}\right| \chi\right\rangle\left._{o}^{A}\right|^{2} \propto \cos ^{2}(\theta-2 \chi) .
$$

To test entanglement we adopt the Clauser-HorneShimony-Holt (CHSH) inequality, given by [23]

$$
S=\left|E(\theta, \chi)-E\left(\theta, \chi^{\prime}\right)+E\left(\theta^{\prime}, \chi\right)+E\left(\theta^{\prime}, \chi^{\prime}\right)\right| \leq 2,
$$

where $E(\theta, \chi)$ is calculated from the $A-B$ photon coincidence counts $C(\theta, \chi)$ according to

$$
E(\theta, \chi)=\frac{C(\theta, \chi)+C\left(\theta+\frac{\pi}{2}, \chi+\frac{\pi}{4}\right)-C\left(\theta+\frac{\pi}{2}, \chi\right)-C\left(\theta, \chi+\frac{\pi}{4}\right)}{C(\theta, \chi)+C\left(\theta+\frac{\pi}{2}, \chi+\frac{\pi}{4}\right)+C\left(\theta+\frac{\pi}{2}, \chi\right)+C\left(\theta, \chi+\frac{\pi}{4}\right)}
$$

Whilst the CHSH inequality is commonly applied to nonlocal measurements on two spatially separated entangled photons, testing for hidden variable theories, here we apply it to single-photon entanglement to test for contextuality. In Fig. 2a the coincidence counts are shown as a function of spatial mode orientation $\chi$ for different values of polarization angles $\theta$. The occurrence of high-
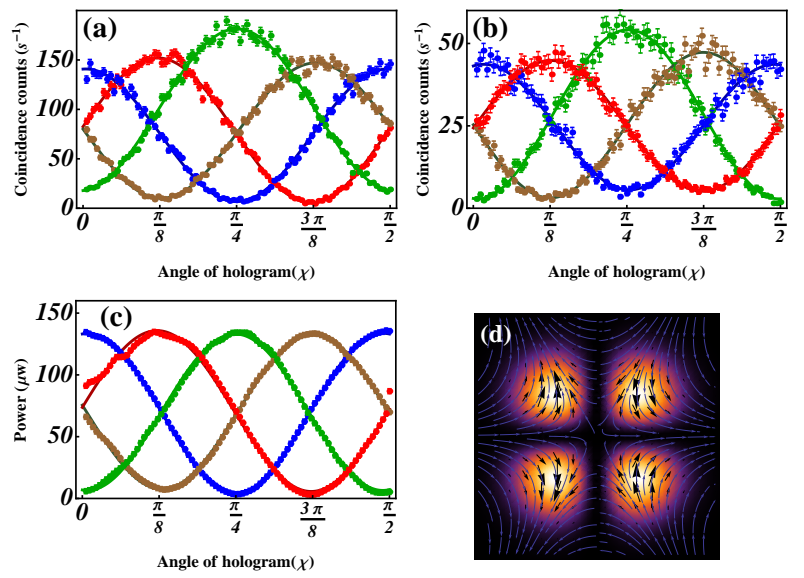

FIG. 2: The experimental coincidence counts as a function of orientation of the sector hologram for different values of polarization direction, for heralded single photons (a), photon pairs (b) and coherent-states (c): blue dots $-\theta=0$, red dots - $\theta=\pi / 4$, green dots $-\theta=2 \pi / 4$, gray dots $-\theta=3 \pi / 4$. The solid lines are the best theoretical fit over the experimental data. The fringe contrast is about $90 \%$, which is much larger than $70.7 \%$, as required for Bell's inequality verification. (d) Simulated intensity and polarization distribution patterns of the optical field for the beam emerging from the $q$-plate in the case of horizontal polarization input beam.

visibility fringes indicates (single-particle) entanglement in the SAM-OAM spaces. The CHSH $S$ value calculated from this data is shown in Fig. 3 (green dots). A violation of the CHSH inequality is clearly obtained, in good agreement with quantum theory predictions, confirming the entanglement and providing a demonstration of quantum SAM-OAM contextuality for single photons.

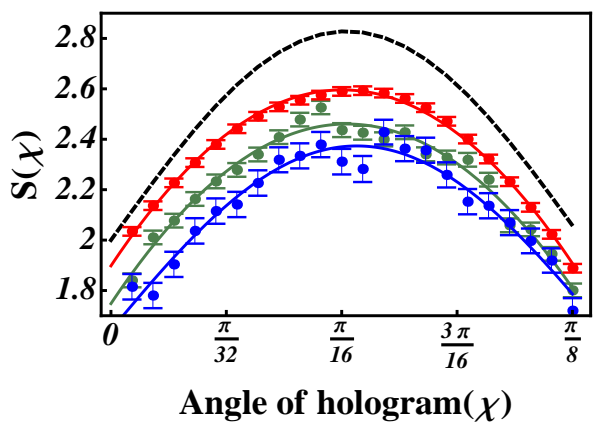

FIG. 3: The CHSH $S$ value in a region where it is larger than the classical limit 2 . The choice of the variables appearing in Eq. (4) is the following: $\theta=0, \theta^{\prime}=\pi / 4, \chi$ is the plot abscissa, $\chi^{\prime}=\chi+\pi / 8$. The green, blue, and red dots correspond to the experimental data in the case of single-photon (a), photon-pairs (b), and classical-wave (c) SAM-OAM experiments, respectively. The dashed line is the quantum mechanical ideal prediction. In the two cases (a) and (b), at $\chi=\pi / 16$, the CHSH inequality is violated respectively by 17 and 10 standard deviations. The classical case (c) is plotted for comparison

\section{Two-photon experiment}

- In this case, we generate and verify entanglement between the SAM of one photon and the OAM of the other, i.e. we demonstrate non-local hybrid entanglement in these two degrees of freedom. To this purpose, the four-sector and uniform holograms of arms $\mathbf{A}$ and $\mathbf{B}$ were swapped, as displayed in the top-right inset of Fig. 1. The $q$-plate in arm $\mathbf{A}$ and the sector hologram in arm $\mathbf{B}$ of the apparatus, together with subsequent coupling into the single-mode fiber before detection, act so as to post-select the photons with $m= \pm 2$ in Eq. (1), i.e. the post-selected initial two-photon state is $|\psi\rangle=\frac{1}{\sqrt{2}} c_{2}\left(|2\rangle_{o}^{A}|-2\rangle_{o}^{B}+|-2\rangle_{o}^{A}|2\rangle_{o}^{B}\right)|H\rangle_{\pi}^{A}|H\rangle_{\pi}^{B}$. The 
photon $A$ passes through the $q$-plate, acting in this case as a OAM-to-SAM transferrer [21], so that the OAM eigenstates $m= \pm 2$ are mapped into $L$ and $R$ polarized photons with $m=0$, respectively. After this process, the photon pair is projected into the nonlocal state

$$
|\phi\rangle_{n l}=\frac{1}{\sqrt{2}}\left(|L\rangle_{\pi}^{A}|+2\rangle_{o}^{B}+|R\rangle_{\pi}^{A}|-2\rangle_{o}^{B}\right)|0\rangle_{o}^{A}|H\rangle_{\pi}^{B}
$$

where the SAM of one photon is maximally entangled with the OAM of the other. Next, the polarization of the $A$ photon is measured by the HWP rotated at angle $\theta / 2$ followed by the polarizer, and the spatial mode of the $B$ photon by the sector hologram rotated at angle $\chi$ followed by coupling in fiber. Well-defined coincidence fringes with visibility up to $90 \%$ are obtained, as shown in Fig. 2b. Repeating the measurements for different angles $\theta$ and $\chi$, the quantity $S$ was evaluated from Eqs. (4) and (5) and the violation of the $\mathrm{CHSH}$ inequality was verified, as shown in Fig. 3 (blue dots). This violation provides a demonstration of SAM-OAM hybrid entanglement and non-locality, for separated photon pairs.

\section{Classical light experiment}

- In our final experiment, we move to a classical regime of non-separable optical modes occupied by many photons, corresponding to coherent quantum states. A $100 \mathrm{~mW}$ frequency-doubled linearly-polarized continuous wave $\mathrm{Nd}: \mathrm{YVO}_{4}$ laser beam is sent in an optical line equal to arm $\mathbf{A}$ of our quantum apparatus, so as to obtain, after the $q$-plate, a coherent state in the SAM-OAM nonseparable mode $\left|\Phi^{+}\right\rangle$given by Eq. (2) [25]. The calculated structure of this mode is shown in Fig. 2d, for a given input polarization. The mode non-separability is evident, as the polarization is spatially non-uniform [16]. The beam polarization is then filtered by the combination of the HWP at angle $\theta$ and polarizer and its spatial mode by the sector hologram rotated at angle $\chi$, as in the single-photon experiment (a). In this case, no trigger is used and the count rates $C(\theta, \chi)$ in Eq. (5) are replaced by average power measurements, corresponding to photon fluxes. When the angles $\theta$ and $\chi$ are changed, high contrast sinusoidal fringes proportional to $\cos ^{2}(\theta-2 \chi)$ were observed in the overall transmitted power fraction, as shown in Fig. 2c. As shown in Fig. 3 (red dots) we note that the classical experiment mimics the results of the single photon experiment. However, the experiment can of course also be interpreted without assuming the existence of photons. In this case, SAM and OAM measurements can be understood just as wave filtering procedures, and no conclusion can be drawn about discrepancies between classical-realistic and quantum behaviour. Nevertheless, providing a classical analog of single-particle entanglement is interesting in itself and may offer the basis for some entirely classical implementations of quantum computational tasks [26].

\section{CONCLUSIONS}

In conclusion, we have demonstrated hybrid entanglement between the spin and the orbital angular momentum of light in two different regimes: single photons and entangled photon pairs. We have reported an additional classical experiment which mimics the quantum result and although the experimental results appear very similar in the three cases, they provide different and complementary insight into the contextual quantum nature of light.

\section{ACKNOWLEDGMENT}

The project PHORBITECH acknowledges the financial support of the Future and Emerging Technologies (FET) programme within the Seventh Framework Programme for Research of the European Commission, under FET-Open grant number 255914.

* Electronic address: karimi@na.infn.it

[1] J. S. Bell, Rev. Mod. Phys. 38, 447 (1966).

[2] X. S. Ma, A. Qarry, J. Kofler, T. Jennewein and A. Zeilinger, Phys. Rev. A 79, 042101 (2009).

[3] L. Neves, G. Lima, A. Delgado and C. Saavedra, Phys. Rev. A 80, 042322 (2009).

[4] S. M. Roy and V. Singh, Phys. Rev. A 48, 3379 (1993).

[5] N. D. Mermin, Rev. Mod. Phys. 65, 803 (1993).

[6] B. H. Liu et al., Phys. Rev. A 80, 044101 (2009).

[7] Y. Hasegawa et al., Nature 425, 45 (2003).

[8] G. Kirchmair et al., Nature 460, 494 (2009).

[9] B. R. Gadway, E. Galvez, and F. DeZela, J. Phys. B 42, 015503 (2009).

[10] R. J. C. Spreeuw, Found. Phys. 28, 361 (1998).

[11] G. Molina-Terriza, J. P. Torres, and L. Torner, Nat. Phys. 3, 305 (2007).

[12] S. Franke-Arnold, L. Allen, and M. Padgett, Laser \& Photon. Rev. 2, 299 (2008).

[13] J. T. Barreiro, T.-C. Wei, and P. G. Kwiat, Nat. Phys. 4, 282 (2008).

[14] E. Nagali et al., Nat. Photon. 3, 720 (2009).

[15] L. Chen and W. She, J. Opt. Soc. Am. B 27, A7 (2009).

[16] C. V. S. Borges, M. Hor-Meyll, J. A. O. Huguenin, and A. Z. Khoury, arXiv:0911.2440v1 (2010).

[17] B. Jack et al., New J. Phys. 11, 10302 (2009).

[18] J. Leach et al., Opt. Express 17, 8287 (2009).

[19] L. Marrucci, C. Manzo, and D. Paparo, Phys. Rev. Lett. 96, 163905 (2006).

[20] L. Marrucci, C. Manzo, D. Paparo, Appl. Phys. Lett. 88, 221102 (2006).

[21] E. Nagali et al., Phys. Rev. Lett. 103, 013601 (2009). 
[22] This spatial state corresponds to a hypergeometricgaussian mode [24] having the same azimuthal profile as a Hermite-Gauss mode $(1,1)$ rotated at angle $\chi$ with respect to the horizontal plane.

[23] J. F. Clauser, M. A. Horne, A. Shimony, and R. A. Holt, Phys. Rev. Lett. 23, 880 (1969).

[24] E. Karimi et al., Opt. Lett. 32, 3053 (2007).

[25] We note that, when the classical states are described as coherent quantum states, the state of the non-separable mode $\left|\alpha_{\Phi^{+}}\right\rangle$is found to be decomposable in the direct product of the coherent states of the two SAM-OAM eigenmodes $\left|\frac{1}{\sqrt{2}} \alpha_{(R, 2)}\right\rangle$ and $\left|\frac{1}{\sqrt{2}} \alpha_{(L,-2)}\right\rangle$, so that no SAMOAM photon entanglement is actually present in the beam [16].

[26] R. J. C. Spreeuw, Phys. Rev. A 63, 062302 (2001). 